\title{
VARICELLA VACCINATION IN A NON-FINANCED PROGRAM
}

Mónica López-Lacort', Eliseo Pastor Villalba², Manuel Valdivia Guijarro3, Cintia Muñoz-Quiles', Alejandro Orrico-Sánchez1, Javier Díez-Domingo'

'Vaccine Research Department, FISABIO-Public Health, Valencia, Spain

2Dirección General de Salud Pública-FISABIO. Conselleria de Sanitat, Valencia, Spain

${ }^{3}$ Hospital Universitario 12 de Octubre, Madrid, Spain

To describe varicella vaccination status in children of the Valencia Region, Spain.

\section{Methods}

A retrospective population-based cohort study using health databases. The study population comprised children of the Valencia Region born between 2006 and 2014 and followed during the study period 2009-2014.

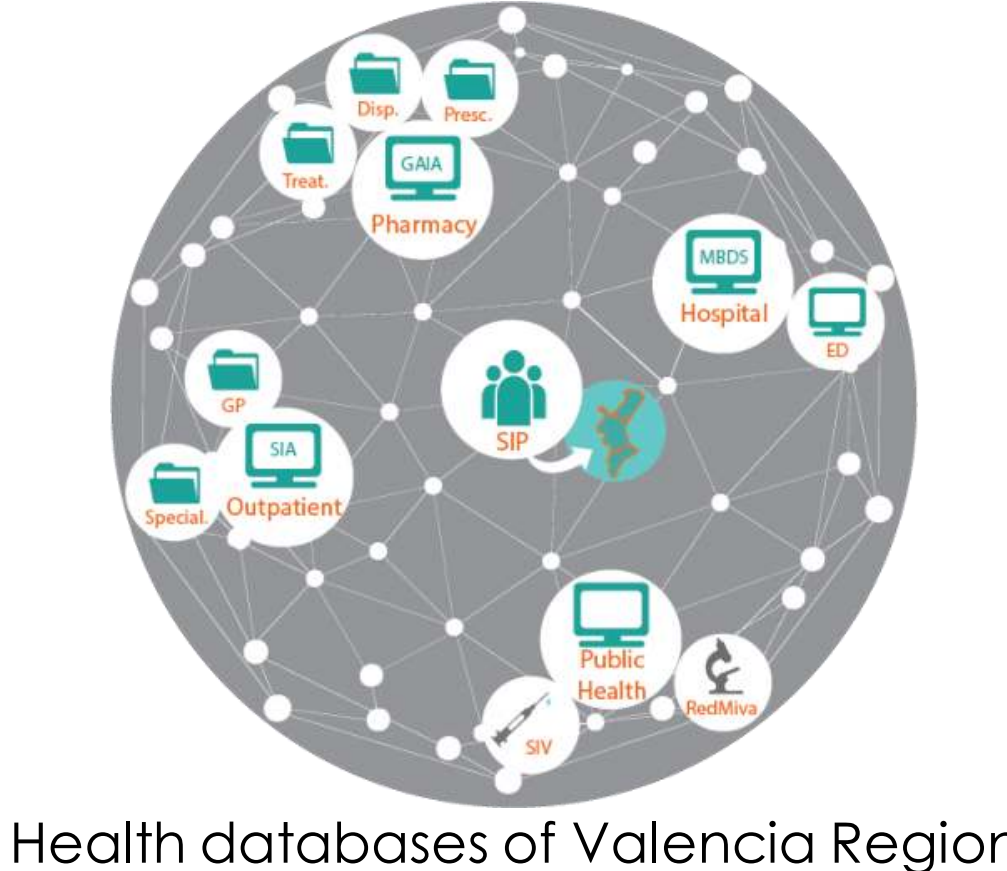

Data sources: population information system (SIP), vaccine information system (SIV) and outpatient information system (SIA) (incident cases of varicella, ICD-9: 052.X were obtained).

Vaccination coverage was estimated by month and year, year of birth, age and health department. Children younger than one year with and without an episode of varicella in the first year of life have been considered to evaluate their vaccination status.
The cohort included 411664 children. Of them, $36.6 \%$ were vaccinated with at least one dose and $\mathbf{1 4 . 3 \%}$ with two doses. Coverage (at least one dose) per cohort ranged $\mathbf{4 2 - 5 1 \%}$ in children born 2006-2011. Subjects born in 2012 and 2013 had lower coverage (32\% and 6\%, respectively).
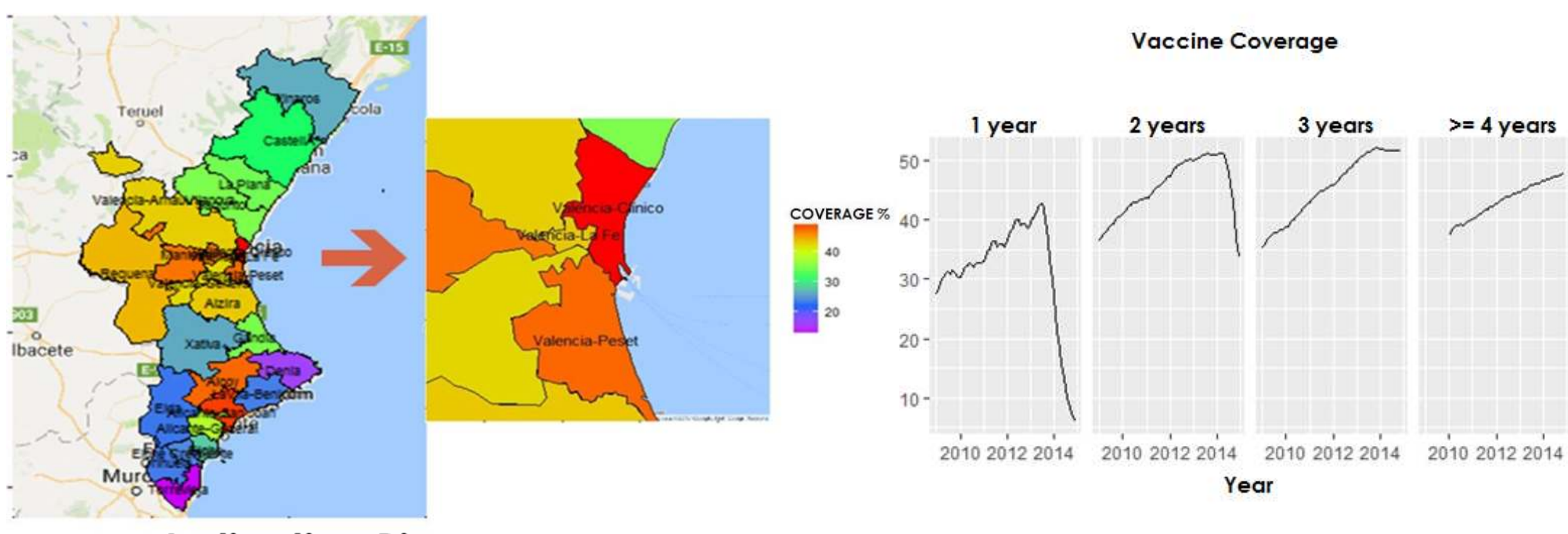

\section{Indication Bias}

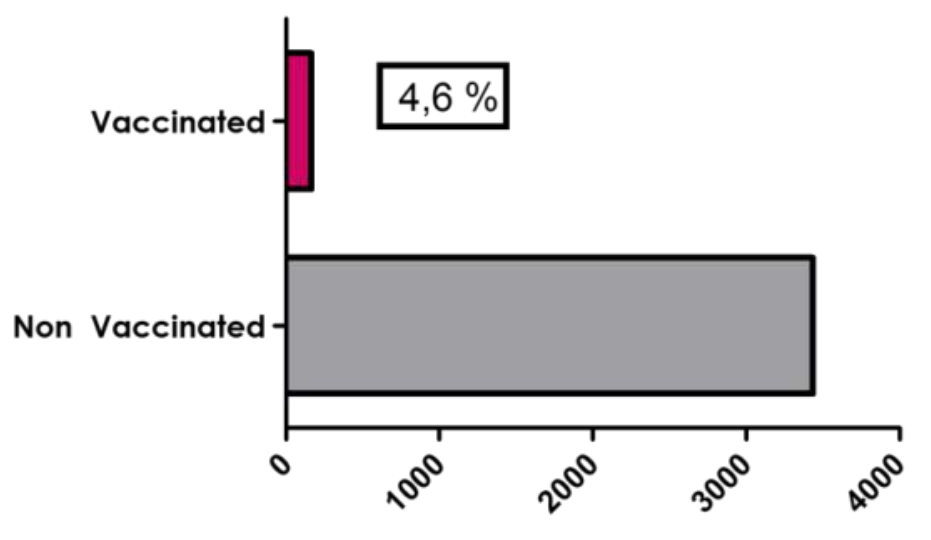

hildren with varicello in
Of 3600 children with a varicella diagnosis during first year of age, only $4.3 \%$ were vaccinated with the first dose during the second year of age, while $33 \%$ of children without varicella during the first year were vaccinated.

\section{Conclusion}

Coverage in the study period was moderate and variable among health departments. There is a temporal pattern marked by the shortage of the vaccine. Although clinical guidelines recommend varicella vaccination even after a varicella episode, we found a clear indication bias. 